Chirurg 2012 $83: 109$

DOI 10.1007/s00104-011-2180-y

Online publiziert: 22. Januar 2012

๑) Springer-Verlag 2012

J. Werner · M.W. Büchler

Klinik für Allgemein-, Viszeral- und Transplantationschirurgie, Universität Heidelberg

\title{
Intraduktale papillär muzinöse Neoplasien des Pankreas
}

IPMNs maligne entarten, wäre eine Differenzierung zwischen IPMNs mit hohem und solchen mit niedrigem Risiko der malignen Transformation wünschenswert. Bisher werden hierfür die im Rahmen einer Konsensuskonferenz in Sendai im Jahre 2006 erarbeiteten Kriterien verwendet.

Man unterscheidet prinzipiell zwischen Hauptgang- und SeitengangIPMNs sowie sog. gemischten Typen. Hauptgang-IPMNs haben ein malignes Entartungsrisiko von ca. 70\% und sollten chirurgisch onkologisch reseziert werden. IPMNs vom Seitengangtyp dagegen entarten in ca. 30\%, sodass ein differenziertes Vorgehen empfohlen wurde. Es wurden aufgrund der damaligen Datenlage morphologische Risikokriterien (u. a. Größe, solide Anteile, Wachstumstendenz) erstellt, die ein höheres Malignitätsrisiko aufzeigen sollten. Die Therapie der Seitengang-IPMNs reicht somit heute von engmaschigen Nachkontrollen und reiner Beobachtung bis hin zu onkologischen Resektionen. Zur Diagnostik und Nachkontrolle wird neben der Schnittbildgebung (Computertomographie, Magnetresonanztomographie mit MR-Cholangiopankreatikographie) vor allem die Endosonographie verwendet. Viele Zentren haben in den letzten Jahren ihre Erfahrung mit den unterschiedlichen diagnostischen und therapeutischen Verfahren publiziert. So werden heute verschiedene histologische IPMN-Subtypen charakterisiert, die eine unterschiedliche Langzeitprognose haben. Trotz erheblicher Fortschritte in der Diagnostik ist allerdings weiterhin unklar, welche Kriterien eine sichere Differenzierung zwischen Läsionen ohne malignes Potenzial und Läsionen mit Progress und maligner Transformation erlauben. Die bisher gültigen Sendai-Kriterien bedürfen jedenfalls einer kritischen Überarbeitung. Somit sollte bei jedem Patienten bei der Entscheidungsfindung der richtigen Therapie zum einen das Malignitätsrisiko bei der reinen Beobachtung einer prämalignen Läsion und zum anderen das Operationsrisiko betrachtet werden.

Der Wissenszuwachs über Klassifikation, Diagnostik und Therapie von IPMNs war in den letzten Jahren erheblich. In dieser Ausgabe wird in vier Beiträgen zu den wichtigsten Aspekten des Themenkomplexes der aktuelle Wissensstand dargestellt und soll Hilfestellung bei dem klinischen Umgang mit IPMNs geben.

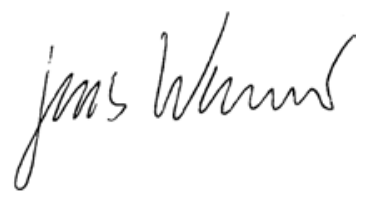

Prof. Dr. J. Werner

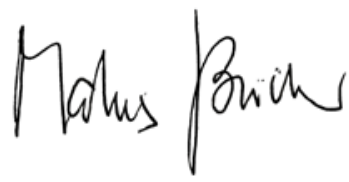

Prof. Dr. M.W. Büchler

\section{Korrespondenzadresse}

Prof. Dr. M.W. Büchler

Klinik für Allgemein-, Viszeral-

und Transplantationschirurgie,

Universität Heidelberg,

Im Neuenheimer Feld 110, 69120 Heidelberg

Markus.Buechler@med.uni-heidelberg.de 